\title{
Children's Mental and Behavioral Health, Schooling, Sociodemographic and Socioeconomic Characteristics During School Closure in France Due To COVID-19: The SAPRIS Project.
}

\section{Maëva Monnier}

Centre Hospitalier Charles Perrens

Flore Moulin

Inserm

Xavier Thierry

National Institute for Demographic Studies

Stéphanie Vandentorren

Santé Publique France

\section{Sylvana Côté}

University of Montreal

\section{Susana Barbosa}

French National Centre for Scientific Research

Bruno Falissard

University of Paris-Saclay

Sabine Plancoulaine

Inserm

\section{Marie-Aline Charles}

Centre for Research in Epidemiology and Statistics

Thierry Simeon

ELFE joint unit

\section{Bertrand Geay}

ELFE joint unit

Laetitia Marchand

EPIPAGE2 joint unit

\section{Pierre-Yves Ancel}

Centre for Research in Epidemiology and Statistics

\section{Maria Melchior}

Pierre Louis Institute of Epidemiology and Public Health

\section{Alexandra Rouquette}

University of Paris-Saclay 
Cédric Galera ( $\square$ cedric.galera@u-bordeaux.fr)

University of Bordeaux

\section{Research Article}

Keywords: Attention Deficit Disorder with Hyperactivity, Children's health, COVID-19, Emotional Symptoms, Risk factors, School closure

Posted Date: June 17th, 2021

DOl: https://doi.org/10.21203/rs.3.rs-568392/v1

License: (c) (1) This work is licensed under a Creative Commons Attribution 4.0 International License. Read Full License

Version of Record: A version of this preprint was published at Scientific Reports on November 17th, 2021. See the published version at https://doi.org/10.1038/s41598-021-01676-7. 


\section{Abstract}

Background: COVID-19 limitation strategies have led to widespread school closures around the world. The present study reports children's mental health and associated factors during the COVID-19 school closure in France in the spring of 2020.

Methods: We conducted a cross-sectional analysis using data from the SAPRIS project set up during the COVID-19 pandemic in France. Using multinomial logistic regression models, we estimated associations between children's mental health, children's health behaviors, schooling, and sociodemographic and socioeconomic characteristics of the children's families.

Results: The sample consisted of 5702 children aged 8 to 9 years, including $50.2 \%$ girls. In multivariate logistic regression models, children's sleeping difficulties were associated with children's abnormal hyperactivity-inattention (adjusted Odds Ratio (aOR) 2.05; 95\% Confidence Interval 1.70-2.47) and emotional symptoms (aOR 5.34; 95\% Cl 4.16-6.86). Factors specifically associated with abnormal hyperactivity/inattention were: male sex (aOR 2.29; 95\% Cl 1.90-2.76), access to specialized care prior to the pandemic and its suspension during school closure (aOR 1.51; 95\% $\mathrm{Cl} 1.21-1.88$ ), abnormal emotional symptoms (aOR 4.06; 95\% $\mathrm{Cl} 3.11-5.29)$, being unschooled or schooled with assistance before lockdown (aOR 2.13; 95\% Cl 1.43-3.17), and tutoring with difficulties or absence of a tutor (aOR 3.25; 95\% $\mathrm{Cl} 2.64-3.99$; aOR 2.47; 95\% Cl 1.48-4.11, respectively). Factors associated with children's emotional symptoms were the following: being born pre-term (aOR 1.34; 95\% Cl 1.03-1.73), COVID-19 cases among household members (aOR 1.72; 95\% Cl 1.08-2.73), abnormal symptoms of hyperactivity/inattention (aOR 4.18; 95\% Cl 3.27-5.34) and modest income (aOR 1.45; 95\% Cl 1.07-1.96; aOR 1.36; 95\% Cl 1.01-1.84).

Conclusions: Multiple characteristics were associated with elevated levels of symptoms of hyperactivityinattention and emotional symptoms in children during the period of school closure due to COVID-19. Further studies are needed to help policymakers to balance the pros and cons of closing schools, taking into consideration the educational and psychological consequences for children.

\section{Introduction}

The emergence of COVID-19 in 2020 led many countries to implement strict sanitary measures, many of which resulted in substantial changes in children's lifestyles for several months. On March 31, 2020, 170 countries temporarily closed schools in an attempt to limit the spread of the new coronavirus responsible for this disease (1). In France, the first lockdown lasted from March 16 to May 11, 2020, and schools and universities, which offer teaching to 14 million students, closed from the beginning of the lockdown until June 22, 2020 (2). School closures, the absence of usual sports and leisure activities, and a reduction in social contacts may have impacted children's mental health in an unprecedented manner (3-9). Children may have been exposed to stressors generated by the media, the fear of contagion, the lack of social interactions with individuals outside the immediate family, and by family members affected with COVID$19(10,11)$. Moreover, the COVID-19 crisis may have resulted in socioeconomic difficulties (e.g., financial 
loss and changes in employment status) and impacted the mental health of adults, which in turn may have had indirect effects on mental health in children $(10,12,13)$. The potential benefits of closing schools to curb the spread of COVID-19 need to be weighed against the effects on children's mental health (14).

Although there is no consensus on the effectiveness of school closures to minimize the spread of COVID19 (14-16), deleterious short-term effects on children's mental health have been observed. A Chinese study of 7143 college students found that $24.9 \%$ experienced anxiety related to the COVID-19 outbreak (3). Another Chinese cross-sectional study using an online questionnaire on 359 children and 3254 adolescents showed that $22.3 \%$ suffered from depressive symptoms during the outbreak (17). In addition, an internet-based survey evaluated the behavioral problems of 1264 children using the Strengths and Difficulties Questionnaire (SDQ), in two elementary schools in China during the COVID-19 outbreak (18). The prevalence was $6.3 \%$ for hyperactivity/inattention and $4.7 \%$ for emotional symptoms (18). However, to date the majority of studies on this topic have been carried out in China where the epidemic has been of shorter duration than in other parts of the world $(3,4,17,19-21)$. In France, a survey that used the SDQ on 432 parents showed that $24.7 \%$ of their children had hyperactivity/inattention symptoms and that $7.1 \%$ had emotional difficulties during the first lockdown in the spring of 2020 (13). This study based on a small sample of children identified significant correlates of these symptoms with parental mental health or financial difficulties, as well as the child's sleeping difficulties or high screen time use. Therefore, social inequalities related to children's health were exacerbated by this unprecedented situation (5).

These studies had several limitations like a small sample size or the non-inclusion of other factors that might affect children's mental health, such as chronic illness and number of hours of study at home per day (22). Furthermore, while a significant number of studies were conducted on the indirect effects of the COVID-19 pandemic on mental health, only a few focused on children $(12,23)$. More research is therefore needed to quantify the impact of socioeconomic disparities and inequalities that are exacerbated by school closure and that of the lockdown on children's mental health (24). The present study sought to assess the factors associated with the mental health of children aged 8 to 9 years-old during the school closure in the first lockdown in spring of 2020 in France. We focused on children's hyperactivity/inattention and emotional symptoms, and on a range of factors such as health behaviors (e.g., sleeping difficulties), schooling (e.g., tutoring, time devoted to schoolwork), and sociodemographic and socioeconomic characteristics of children's families (e.g., financial difficulties, type of housing, occupational category).

\section{Methods}

In the early weeks of the COVID-19 crisis, the SAPRIS survey was set up to study the health, social interactions and socioeconomic characteristics of the general population during the crisis in France (25). The project was based on the collection of data using the same questionnaire on the participants of five large ongoing French cohorts, three of adults (Constances, E3N-E4N and NutriNet-Santé) and two of children (ELFE/EPIPAGE-2). For the present study, we used data collected on the 5702 children aged 8-9 
years old in 2020 and participating in the ELFE and EPIPAGE-2 population-based birth cohorts that focus on child health and include late pre-term and term (ELFE) and very and extremely premature children (EPIPAGE-2) born in 2011 (26-28). More details on ELFE and EPIPAGE 2 are available elsewhere (26-28). Data for the present study were reported by parents during the first lockdown period (from April 16 to May 4,2020 ) or immediately after (from May 5 to June 21, 2020), thus encompassing the whole period of school closure (29). To make the target populations completely disjointed and complementary, 48 children born before 35 weeks in ELFE were excluded. All methods were performed in accordance with the relevant guidelines and regulations. Ethical approval and written informed consent were obtained from each participant before enrolment in the original cohort. The study was approved by the Inserm ethics evaluation committee ( $\mathrm{n}^{\circ}$ 2020.04.24 bis_ 20.04.22.74247, 2020 April 27), and the CNIL ( $\left(n^{\circ} 920193\right.$, 2020 April 30). According to French law, the present nested survey did not require specific additional written consent from the participant. Representatives of the participants tested and validated the questionnaires, but they did not contribute to other aspects related to the design, conduct, reporting or dissemination of the research.

\section{Children's mental health}

Symptoms of hyperactivity/inattention and emotional symptoms were ascertained by two subscales of the SDQ, a widely used measure of children's mental health which has satisfactory psychometric properties $(30,31)$. These symptoms concerned the 15 days prior to the questionnaire and were reported by parents on a 3-point scale (not true, somewhat true, and certainly true; ranges from $0-2$ ) to indicate the extent to which each item applied to their children $(32,33)$. The following five items were used to assess symptoms of hyperactivity/inattention: "Restless, overactive, cannot stay still for long"; "Constantly fidgeting or squirming"; "Easily distracted, concentration wanders"; "Thinks things out before acting"; and "Sees tasks through to the end, good attention span" (32). The five items used to assess the emotional symptoms were: "Complains of headache/stomach ache"; "Many worries, often seems worried"; "Often unhappy, down-hearted or tearful"; "Nervous or clingy in new situations, easily loses confidence"; and "Many fears, easily scared" (32). From the parents' responses to the five items of each subscale, we calculated scores that ranged from 0 to 10 with cut-offs of the French version of the SDQ (34). Concerning hyperactivity/inattention, a score $\leq 5$ is considered normal, equal to 6 as boundary state, and $>6$ as abnormal. For emotional symptoms, a score $\leq 3$ is considered normal, equal to 4 as boundary state, and $>4$ as abnormal.

\section{Covariates}

\section{Children's health behavior}

Health behaviors included the following: sleeping difficulties (e.g., difficulty falling asleep, waking up frequently or too early at night without being able to go back to sleep) since the beginning of lockdown (yes: new occurrence, increase, or stability, no: decrease, disappearance or none); time per day spent by child on the following activities: (a) reading, drawing, board games; (b) screen for recreation, (c) social network, (d) physical activity (sports or walks outside; indoor and outdoor physical activity). 


\section{Children's schooling}

Educational characteristics included the following: school situation before lockdown (normal; with assistance or unschooled); average time devoted to schoolwork per day (none or less than one hour, 1-3 hours, > 3 hours); tutoring difficulty (tutoring with difficulty, tutoring without difficulty, no tutoring); average screen time for educational reasons per day. Tutoring refers to the presence of someone who can help the child with homework (e.g., parents, siblings, grandparents etc.). Screen time for educational reasons refers to time spent on television or other screens to follow school or educational programs.

\section{Sociodemographic and socioeconomic characteristics of children's families}

Socioeconomic characteristics included the following: the parents' occupational category (executive, intermediate and executive, intermediate and employee, independent, laborer, 2 inactive or only one employee/laborer); change in parents' work situation (change at least for one parent, no change for either parent); parents' distance working (neither working, one teleworking and the other not working, at least one working outside, both teleworking); perceived financial situation (affluent and constant income; affluent and declining income; modest and constant income; modest and declining income). Sociodemographic information concerning housing conditions included the following: type of housing (rural house, urban house, flat with balcony/garden, flat without balcony/garden, other); region classified according to the prevalence of COVID-19 at the beginning of the epidemic (High: lle de France and Grand Est vs Medium: Bourgogne-Franche-Comté, Auvergne-Rhône-Alpes and Hauts-de-France vs Low: other regions); number of rooms per inhabitant and if the child lived with both parents or not.

\section{Others}

Additional data

included the following: sex; chronic disease (yes, no); born pre-term (yes, no); children's access to specialized care prior to the COVID-19 epidemic (e.g., physiotherapist, speech therapist, psychologist, rehabilitation) and its continuation during school closures (yes and pursuit, yes but no pursuit, no); COVID-19 cases among household members (yes, no).

\section{Statistical analysis}

We first described child and family sociodemographic characteristics, (i.e., weighted means with standard deviations for continuous variables; frequency with weighted percentages for categorical variables). Descriptive analyses were conducted after correcting the sample data to be representative of children born in France in 2011 (35). For this purpose, a weighting coefficient was calculated. For more information, see the online supplement.

Before performing multiple imputation, we removed variables with more than $30 \%$ missing data (screen time and physical activity) and observations with missing data for both outcomes (hyperactivity/inattention and emotional symptoms). Classification and Regression Tree methods (CART, imputation method for mixed data: both Continuous and Categorical) (36) for multiple imputation were used to handle missing data. Secondly, to test the association between each covariate and mental health 
outcomes (i.e., hyperactivity/inattention and emotional symptoms), we used multinomial logistic regressions adjusted on prematurity to estimate Odds Ratios (OR) and 95\% confidence intervals (Cl). Thirdly, to perform relevant covariate selection, we applied a penalized regression method, ElasticNet, combining bootstrapping with multiple imputed data (37-39). We used the variable inclusion probability with a $50 \%$ threshold to select variables associated with children's mental health. Finally, we used multinomial logistic regression to estimate adjusted $\mathrm{OR}$ and $95 \% \mathrm{Cl}$ for the association between variables selected by Elastic-Net, prematurity and both mental health outcomes.

Data were analyzed using the nnet package in $R$ (version 3.6.1) with multinomial logistic regressions specified using the multinom function. The mice package and its cart method were used to perform multiple imputation. Finally, the caret, glmnet, doParallel and $d p l y r$ packages were applied to implement the Elastic-Net method on imputed data.

\section{Results}

\section{Sample characteristics}

The study included 5702 children (Table 1). In total, 2808 (50.2\%) children were females, 1161 (19.4\%) had access to specialized care prior to the COVID-19 epidemic, 289 (4.5\%) had chronic disease, and 1122 (2.9\%) were premature children. COVID-19 cases were reported in $235(4.4 \%)$ households. Concerning the children's mental health, hyperactivity/inattention scores were elevated for $672(13.6 \%)$ and moderate for 452 (8.2\%) children, respectively. Emotional difficulties scores were elevated or moderate for 404 (7.5\%) and $288(5.8 \%)$ children, respectively. In terms of children's health behaviors, $39.9 \%$ had sleeping difficulties. Children spent an average of 2 hours and 20 min reading, drawing, and playing board games per day, and 2 hours and 45 min doing physical activities per day. Regarding screen time, children spent an average of 3 hours and 10 min per day for recreation, and 5 min on social networks. Only 188 (2.8\%) children were unschooled or in school with assistance before the lockdown, 2764 (62.8\%) spent between 1-3 hours per day on schoolwork and an average of 50 minutes on educational programs per day. 4202 $(96.7 \%)$ children had a tutor at home to help them with their homework.

As shown in Table 2, the pandemic situation led to a change in work situation in 1483 (43.9\%) households, working from home was implemented in 2371 households (32.6\%) and 1813 (41.6\%) perceived financial difficulties. Children lived mostly in a house (67.4\%) and with their two parents (84.5\%). $9.0 \%$ and $17.3 \%$ of children lived in regions with high or moderate prevalence of COVID, respectively.

\section{Factors associated with hyperactivity/inattention and/or emotional symptoms}

The factors associated with hyperactivity/inattention and/or emotional symptoms when adjusting for prematurity are presented in Tables 3 and 4 . The factors associated with an increased risk of both hyperactivity/inattention and emotional symptoms in univariate analysis were the following: having chronic disease, being born pre-term, having access to specialized care prior to the COVID-19 epidemic, 
having difficulties in sleeping, being schooled with assistance or unschooled before the lockdown, having difficulties with tutoring or not having a tutor, low parents' dominant socio-professional category, presence of change in the parents' work situation, having modest and declining outcome during the lockdown, and not living with both parents. The factor associated with a lower risk of both hyperactivity/inattention and emotional symptoms adjusted on prematurity was having at least one parent who worked outside.

Factors exclusively associated with an increased risk of hyperactivity/inattention adjusted on prematurity were the following: being a boy; having emotional symptoms. Factors associated with a decreased risk of symptoms of hyperactivity/inattention were the following: spending 1-3 hours or more than 3 hours per day devoted to schoolwork, having parents working remotely, and living in an urban environment.

In contrast, factors only associated with an increased risk of emotional symptoms adjusted on prematurity were the following: presence of COVID-19 cases in the household, hyperactivity/inattention symptoms, having modest and constant income, and living in an urban flat without a balcony/garden. The only factor associated with a lower risk of emotional symptoms was being a boy.

Finally, the number of rooms per inhabitant and the French regions most affected by the COVID-19 epidemic were not associated with children's mental health.

\section{Factors associated with children's mental health after Elastic Net selection}

The factors most strongly associated with children's mental health after using Elastic Net selection are presented in Tables 5 and 6. Elastic Net selected 10 factors as most associated with hyperactivity/inattention in children: sex, access to specialized care prior to the COVID-19 epidemic, having emotional symptoms, having sleeping difficulties, being schooled with assistance or unschooled before the lockdown, devoting one hour or less to school work per day, having difficulties with tutoring or not having a tutor, low parents' dominant socio-professional category, living in an urban house, and not living with both parents (Table 5).

After adjusting for these 10 factors most associated with hyperactivity/inattention symptoms and prematurity, boys remained at greater risk for abnormal hyperactivity-inattention than girls (aOR 2.29; $95 \%$ $\mathrm{Cl}$ 1.90-2.76). Children who had regular care before the COVID-19 epidemic and could not continue the sessions during the lockdown had an increased risk of abnormal hyperactivity/inattention symptoms (aOR 1.51; 95\% Cl 1.21-1.88).

Concerning children's mental health, compared to normal children, children with abnormal emotional symptoms or at boundary state were associated with higher risk of abnormal hyperactivity/inattention symptoms (aOR 4.06; 95\% Cl 3.11-5.29 and aOR 2.14; 95\% Cl 1.54-2.97, respectively). Regarding the children's health behavior, risk of abnormal hyperactivity/inattention symptoms or at boundary state appeared to be higher for children with sleeping difficulties (OR 2.05; 95\% $\mathrm{Cl} 1.70-2.47, \mathrm{OR} 1.71 ; 95 \% \mathrm{Cl}$ 1.39-2.10) than in those without sleeping difficulties (Table 5). 
Regarding children's schooling, being schooled with assistance or unschooled was associated with a higher risk of abnormal hyperactivity/inattention (aOR 2.13; 95\% Cl 1.43-3.17). Spending 1-3 hours per day devoted to schoolwork or more than 3 hours seemed to be associated with less risk of abnormal hyperactivity/inattention symptoms than spending no time or less than 1 hour (aOR 0.55 ; 95\% Cl 0.340.91 and aOR $0.56 ; 95 \% \mathrm{Cl} 0.33-0.92$, respectively). In this multivariate analysis, having difficulties with tutoring or not having a tutor were still associated with a higher risk of abnormal hyperactivity/inattention (aOR 3.25; 95\% Cl 2.64-3.99 and aOR 2.47; 95\% Cl 1.48-4.11, respectively).

For sociodemographic and socioeconomic characteristics, the low parents' occupational category (laborer) was still associated with a higher risk of abnormal hyperactivity/inattention as compared to the executive category (aOR 1.66; $95 \% \mathrm{Cl} 1.01-2.71$ ). Compared to living in a rural house, living in an urban house was associated with less risk of abnormal hyperactivity/inattention symptoms (aOR 0.79; $95 \% \mathrm{Cl}$ 0.64-0.97).

Concerning the factors most associated with emotional symptoms, Elastic Net selected the following seven variables: being born pre-term, COVID-19 cases in the household, hyperactivity-inattention symptoms, sleeping difficulties, parents' occupational category, change in parents' work situation, and financial situation (Table 6). The results described below are adjusted on these seven variables.

Children born pre-term remained at a higher risk of abnormal emotional symptoms (aOR $1.34 ; 95 \% \mathrm{Cl}$ 1.03-1.73). The presence of COVID-19 cases in the household (aOR 1.72; 95\% Cl 1.08-2.73), abnormal symptoms of hyperactivity/inattention (aOR 4.18; 95\% Cl 3.27-5.34) and sleeping difficulties (aOR 5.34; $95 \% \mathrm{Cl} 4.16-6.86$ ) remained risk factors of abnormal emotional symptoms (Table 6). In this multivariate analysis, only the "intermediate and executive" parents' occupational category was still associated with a higher risk of abnormal emotional symptoms as compared to the "executive" category (aOR $1.61 ; 95 \% \mathrm{Cl}$ 1.16-2.21). Having a modest income remained a risk factor of abnormal emotional symptoms (aOR 1.45; $95 \% \mathrm{Cl} 1.07-1.96 ;$ aOR $1.36 ; 95 \% \mathrm{Cl} 1.01-1.84$ ). A change in parents' work situation during school closures was no longer significantly associated with emotional symptoms.

\section{Discussion}

\section{Main findings and interpretations}

Our study showed high rates of hyperactivity/inattention and emotional symptoms during school closure due to COVID-19 in France. It also helped in identifying risk factors of hyperactivity/inattention and/or emotional symptoms in children. Difficulties in sleeping and parents' occupational category were the only factors independently associated in the multivariate models with both dimensions of mental disorder. Factors specifically associated with hyperactivity/inattention were the following: sex, presence of regular care and its pursuit during school closure, emotional symptoms, school situation, presence of tutoring and difficulties with it, and type of housing. Factors related to emotional symptoms were the following: being born pre-term, the presence of COVID-19 cases in the household, hyperactivity/inattention and financial difficulties. 


\section{Children's sex, chronic disease, specialized care and being born pre-term}

In the context of the COVID-19 crisis, our results indicate a higher risk of hyperactivity/inattention among boys than girls, and a higher risk of emotional symptoms among girls than boys. Findings by others were similar outside the context of the COVID-19 pandemic $(40,41)$ and our findings are consistent with those of recent studies on it $(4,42,43)$. A cross-sectional online survey of 8079 Chinese adolescents aged 12-18 affected by the COVID-19 pandemic revealed that female gender was the most important risk factor for depressive and anxiety symptoms (4).

After adjustment on covariates, we found that children who had access to specialized care prior to the COVID-19 pandemic and who were unable to continue follow up during school closures had a higher risk of abnormal hyperactivity-inattention. Being born pre-term was associated with a higher risk of abnormal emotional symptoms during the COVID-19 pandemic. These results are coherent with epidemiological studies conducted outside the COVID-19 context which found that pre-and perinatal factors (e.g. prematurity, chronic disease) are associated with Attention-Deficit/Hyperactivity Disorder (ADHD) (44$46)$ and emotional problems $(47,48)$, although the definite causes remain unknown. While being born preterm is a known risk factor for hyperactivity/inattention disorder $(49,50)$, it is the association between prematurity and emotional symptoms that is emphasized by our findings.

\section{COVID-19 cases in the household}

In our multivariate analyses, the presence of COVID-19 cases in the household seemed to be a risk factor of abnormal emotional symptoms in children. This was one of the strongest associations according to Elastic Net. These results are in line with a large cross-sectional online survey of 44447 college students conducted in China (51). Compared with students who reported not having infected or suspected cases in family members and relatives, those who reported having confirmed and suspected cases in family members and relatives had a higher risk of depressive symptoms (OR 4.06; $95 \% \mathrm{Cl} 1.62-$ 10.19 and OR $2.11 ; 95 \% \mathrm{Cl} 1.11-4.00$, respectively) (51). Interestingly, several studies evidenced an association between emotional symptoms and COVID-19 epidemic-related factors such as potential exposure to the virus, fear of infection (i.e., self- and/or family members), and loss of loved ones $(3,52,53)$. Mental stress, anxiety, depressive symptoms, insomnia and fear are triggered by the pandemic itself, mandatory preventive measures, and COVID-19-related circumstances (52-54).

\section{Children's mental health}

Our findings showed that during the school closure in France, hyperactivity/inattention and emotional symptoms were associated. Elastic Net found this to be one of the strongest associations. Our results confirm those of another study conducted outside the epidemic context which showed a stronger correlation between hyperactivity/inattention and emotional symptoms in the SDQ (33).

\section{Children's health behavior: sleep difficulties}


Our study is consistent with recent reports on the association between difficulties in sleeping and children's mental health during the COVID-19 pandemic (13), in particular with hyperactivity/inattention and emotional symptoms. Difficulty in sleeping is one of the most predictive variables of hyperactivity/inattention in children, as well as emotional symptoms. Our findings confirm those of previous studies conducted outside the COVID-19 pandemic (55-57). Hyperactivity/inattention and emotional symptoms are both characteristics of children with ADHD. A meta-analysis of 16 studies comparing sleep in ADHD children versus controls found that the former were significantly more impaired than the latter on most subjective and some objective sleep measures (57). The relationship between ADHD and difficulties in sleeping is bidirectional (56,58-60). ADHD may cause sleep problems and sleep problems may cause or mimic ADHD $(55,56)$. Moreover, a reciprocal relationship between sleep quality and anxiety problems seems very likely, although the specific mechanisms that contribute to these sleep disturbances remain unclear (61-64).

\section{Children's schooling: school situation, time devoted to schoolwork, and tutoring}

In multivariate analyses, being unschooled or schooled with assistance before the lockdown was associated with a higher risk of abnormal hyperactivity/inattention. Spending 1-3 hours or more than 3 hours per day on schoolwork seemed to be associated with a lower risk of abnormal hyperactivity/inattention symptoms than spending no time or less than 1 hour. Furthermore, children without a tutor and those with tutoring difficulties were at greater risk for abnormal hyperactivityinattention compared with those without tutoring difficulties. Our results are consistent with those of a previous study, which found that prolonged school closure regardless of the epidemic context can cause significant mental health problems in children, especially among economically disadvantaged groups (65). While schools are essential for children's academic education, they also play an important role in addressing their physiological and mental health needs $(65,66)$. However, according to Elastic Net, these schooling variables are not strongly associated with emotional symptoms.

\section{Sociodemographic and socioeconomic characteristics of children's families}

Our multivariate analyses found an increased risk of abnormal emotional symptoms in children whose families experienced financial difficulties. Children of parents in a low socio-professional category were also the most affected in terms of hyperactivity/inattention. Similar results were recently reported in a French community-based sample of 432 parents and their children during the COVID-19 lockdown (13). The authors showed that family socioeconomic factors such as financial difficulties and unemployed parents were significantly associated with a higher risk of children's mental health problems, such as hyperactivity/inattention and emotional symptoms (13). Stability of family income was significantly associated with children's psychological difficulties during the COVID 19 pandemic (3), due to economic decline and the stress that it causes $(67,68)$.

\section{Housing}


Our adjusted analysis showed that, compared to living in a rural house, living in an urban house was associated with a lower risk of hyperactivity/inattention during school closure in France. The type of housing was not associated with emotional symptoms in multivariate analysis after an Elastic Net selection. These results are contrary to most studies showing a lower risk of ADHD in children living in rural rather than urban areas $(69,70)$. A large web-based survey of 8177 Italian students found that poor housing (i.e., living in apartments $<60 \mathrm{~m}^{2}$ with poor views and poor indoor quality) was associated with an increased risk of depressive symptoms during lockdown (71). In addition, an Iranian study which evaluated people's preferences and priorities to choose healthy homes after the COVID-19 pandemic revealed that the most critical priorities for residents were natural light, having a view, acoustic protection, and an open or semi-open space (72). Our conflicting results may be explained by the greater proximity to other children in urban than in rural areas.

Contrary to findings in the literature (17), our adjusted analysis did not find a significant association between living in one of the regions of France most affected by COVID 19 and children's mental health. Finally, living with both parents was found to be protective for hyperactivity/inattention but not for emotional symptoms. Living with both parents has been shown to be a protective factor for children's mental health in the context of the COVID-19 pandemic, since a Chinese study of 7143 college students found that living with parents was a protective factor against anxiety (OR $0.75 ; 95 \% \mathrm{Cl} 0.60-0.95)(3)$. A recent study on the mental health of children aged 9 to 18 in France also showed the protective effect of a two-parent family on the psychological distress of children during the COVID-19 pandemic (73).

\section{Strengths and limitations}

This study has some limitations. First, owing to too many missing data regarding screen time and physical activity, we were unable to estimate the associations between these variables and the presence of hyperactivity/inattention or emotional symptoms in children. The results could be different or even more marked in the general population. Second, only a few parents reported their alcohol and smoking consumption as well as their mental disorders, which prevented us from taking these variables into account in our analyses. Third, we did not have any objective measures of sleep quality but only a parent's self-reported measure of difficulty sleeping. Fourth, we had no data on the consumption of media information related to the pandemic in minutes per day and quality of information received. Therefore, we were not able to study the association between these environmental factors with children's mental health, even though significant associations have already been reported $(43,52,74)$. Fifth, our data were collected during a period of school closure in the first COVID-19 wave in France, so we could not analyze data on children's mental health prior to the lockdown. As a result, we were unable to assess the impact of school closure on children's mental health independently from that of the COVID-19 pandemic. Sixth, we could not re-evaluate the mental health of the children, yet it is possible that the reopening of schools in France has resulted in health benefits for them. Future study designs should include re-assessment of mental health and compare children's health scores between countries that implemented vs. did not implement school closure as a preventive measure. Future research should also investigate potential environmental factors that might influence children's mental health, such as parental history of mental illness, 
consumption of media information related to COVID-19, and domestic violence. In addition, the causal relationship between environmental factors related to COVID-19 and children's mental health should be explored.

The study also has some strengths. First, it includes the large number of parent respondents during school closures in France at the beginning of the COVID 19 pandemic. It also includes pre-term children, a population more at risk for hyperactivity/inattention, so it provides more power for studying risk and protective factors in a lockdown situation. Second, we were able to control a wide range of possible covariates from sociodemographic and socioeconomic characteristics of children's families to children's schooling. To our knowledge, this is the first study to consider a set of variables related to children's housing and schooling. Third, using validated measures of hyperactivity/inattention and emotional symptoms (SDQ) with satisfactory psychometric properties is a strength and novel aspect of this study $(30,33)$. Overall, we believe that our findings contribute to the evolving debate concerning school closure and children's mental health. We suggest that school closure due to COVID-19 could increase the risk of mental health problems and exacerbate health inequalities (68). Our findings provide important guidance for the development of children's psychological support strategies in France.

\section{Implications}

The current focus on measures to contain COVID-19 transmission around the world may distract attention from emerging mental health issues $(54,75)$. The closure of schools due to COVID-19 and the lockdown could lead to an increase in mental disorders in children, especially among vulnerable populations. Efforts must be made to address the pandemic situation while considering children's mental health and to adapt the delivery of care to meet the demands of COVID-19 (68). Our findings have important clinical and policy implications. In this global public health emergency, identifying vulnerable groups of children and environmental risk factors of children's mental health is a critical step in developing and adapting mental health services and tools (76). Health authorities and governments should implement services that improve the mental health of the population, particularly that of vulnerable children. Emerging digital applications such as telehealth, social media, and mobile health could serve to promote children's mental health (77).

\section{Conclusion}

This study conducted in France explored several risk factors of hyperactivity/inattention and emotional symptoms in children during the school closure due to the COVID-19 pandemic. Some factors that may increase the risk of children developing mental health problems are described: sex, pre-existing health disorders, COVID cases in the household, difficulties in sleeping and in schooling, and sociodemographic and socioeconomic disparities. Policymakers need to balance the pros and cons of reopening school, taking into consideration the educational and psychological consequences for children. Therefore, it is essential to implement appropriate protective measures to manage the unprecedented repercussions of school closure on children's mental health while avoiding an increase in socio-economic inequalities. 


\section{Abbreviations}

ADHD: Attention-Deficit/Hyperactivity Disorder

aOR: Adjusted Odds Ratio

CART: Classification and Regression Tree

Cl: Confidence Intervals

OR: Odds Ratio

SDQ: Strength and Difficulties Questionnaire

VIP: Variable Inclusion Probability

\section{Declarations}

\section{Acknowledgements}

We are grateful to the region of the New-Aquitaine and its AMI FLASH Recherche et Innovations COVID, without whom the project would never have started.

The authors warmly thank all the volunteers of the ELFE, and EPIPAGE2 cohorts.

We thank the staff of the SAPRIS study group that have worked with dedication and engagement to collect and manage the data used for this study and to ensure continuing communication with the cohort participants.

\section{Competing interests}

Mrs Maeva Monnier has been funded by the region of New-Aquitaine (AMI Flash Recherche et Innovations COVID). The other authors declare no competing interests related to this paper.

\section{Fundings}

Role of the funding source

Sponsor and funding sources played no role in the study design, data collection, analysis, interpretation or drafting of the study. MM and CG had full access to all data in the study.

\section{The funders}

ANR (Agence Nationale de la Recherche, 0009/SAPRIS/997/NB), Fondation pour la Recherche Médicale, Direction Générale de la Recherche et de l'Innovation, Institut Gustave Roussy, the New-Aquitaine region. 


\section{Data availability}

In regards to data availability, data of the study are protected under the protection of health data regulation set by the French National Commission on Informatics and Liberty (Commission Nationale de I'Informatique et des Libertés, CNIL). The data can be available upon reasonable request after a consultation with the steering committee of the Sapris study. The French law forbids us to provide free access to Sapris data; access could however be given by the steering committee after legal verification of the use of the data.

\section{References}

1. UNESCO. Education: From disruption to recovery. UNESCO. https://en.unesco.org/covid19/educationresponse (2020).

2. Antona, D. et al. COVID-19 chez l'enfant (moins de 18 ans). État des lieux de la littérature en amont de la réouverture annoncée des crèches et des écoles. État de la littérature au 24 avril 2020. Synthèse rapide COVID-19. Santé Publique France. /import/covid-19-chez-l-enfant-moins-de-18-ans---etat-deslieux-de-la-litterature-en-amont-de-la-reouverture-annoncee-des-creches-et-des-ecoles.-etat-de-I (2020).

3. Cao, W. et al. The psychological impact of the COVID-19 epidemic on college students in China. Psychiatry Res. 287 (2020).

4. Zhou, S.J. et al. Prevalence and socio-demographic correlates of psychological health problems in Chinese adolescents during the outbreak of COVID-19. Eur Child Adolesc Psychiatry. 29(6), 749-758 (2020).

5. Singh, S. et al. Impact of COVID-19 and lockdown on mental health of children and adolescents: A narrative review with recommendations. Psychiatry Res. 24 (2020).

6. Jiao, W.Y. et al. Behavioral and Emotional Disorders in Children during the COVID-19 Epidemic. $J$ Pediatr. 221, 264-266 (2020).

7. Guessoum, S.B. et al. Adolescent psychiatric disorders during the COVID-19 pandemic and lockdown. Psychiatry Res. 291 (2020).

8. Orgilés, M., Morales, A., Delvecchio, E., Mazzeschi, C. \& Espada, J. P. Immediate psychological effects of the COVID-19 quarantine in youth from Italy and Spain. PsyArXiv. Preprint at https://psyarxiv.com/5bpfz/ (2020).

9. Ghosh, R., Dubey, M. J., Chatterjee, S. \& Dubey, S. Impact of COVID -19 on children: special focus on the psychosocial aspect. Minerva Pediatr. 2(3), 226-235 (2020).

10. De Figueiredo, C. S. et al. COVID-19 pandemic impact on children and adolescents' mental health: Biological, environmental, and social factors. Prog Neuropsychopharmacol Biol Psychiatry. 106 (2021).

11. Brooks, S. K. et al. The psychological impact of quarantine and how to reduce it: rapid review of the evidence. Lancet Lond Engl. 395, 912-920 (2020). 
12. Spinelli, M., Lionetti, F., Pastore, M. \& Fasolo, M. Parents' Stress and Children's Psychological Problems in Families Facing the COVID-19 Outbreak in Italy. Front Psychol. https://www.ncbi.nlm.nih.gov/pmc/articles/PMC7350926/ (2020).

13. Moulin, F. et al. Risk and protective factors related to children's symptoms of emotional difficulties and hyperactivity/inattention during the COVID-19-related lockdown in France: results from a community sample. Eur Child Adolesc Psychiatry. https://hal.sorbonne-universite.fr/hal-03165547 (2021).

14. Head, J. R. et al. The effect of school closures and reopening strategies on COVID-19 infection dynamics in the San Francisco Bay Area: a cross-sectional survey and modeling analysis. MedRxiv Prepr Serv Health Sci. figshare https://doi.org/10.1101/2020.08.06.20169797 (2020).

15. Danis, K. et al. Cluster of Coronavirus Disease 2019 (COVID-19) in the French Alps, February 2020. Clin Infect Dis. 71(15), 825-832 (2020).

16. Ludvigsson, J. F. Systematic review of COVID-19 in children shows milder cases and a better prognosis than adults. Acta Paediatr Oslo Nor 1992. 109(6), 1088-1095 (2020).

17. Duan, L. et al. An investigation of mental health status of children and adolescents in china during the outbreak of COVID-19. J Affect Disord. 275, 112-118 (2020).

18. Liu, Q. et al. The prevalence of behavioral problems among school-aged children in home quarantine during the COVID-19 pandemic in china. J Affect Disord. 279, 412-6 (2021).

19. Tang, S., Xiang, M., Cheung, T. \& Xiang, Y. T. Mental Health and Its Correlates among Children and Adolescents during COVID-19 School Closure: The Importance of Parent-Child Discussion. J Affect Disord. https://www.ncbi.nlm.nih.gov/pmc/articles/PMC7550131/ (2020).

20. Xie, X. et al. Mental Health Status Among Children in Home Confinement During the Coronavirus Disease 2019 Outbreak in Hubei Province, China. JAMA Pediatr. 174(9), 898 (2020).

21. Liu, N. et al. Prevalence and predictors of PTSS during COVID-19 outbreak in China hardest-hit areas: Gender differences matter. Psychiatry Res. 287, 112921 (2020).

22. Wiguna, T. et al. Brief Research Report on Adolescent Mental Well-Being and School Closures During the COVID-19 Pandemic in Indonesia. Front Psychiatry. https://www.ncbi.nlm.nih.gov/pmc/articles/PMC7704451/ (2020).

23. Vindegaard, N. \& Benros, M. E. COVID-19 pandemic and mental health consequences: Systematic review of the current evidence. Brain Behav Immun. 89, 531-542 (2020).

24. Viner, R. M. et al. School closure and management practices during coronavirus outbreaks including COVID-19: a rapid systematic review. Lancet Child Adolesc Health. 4(5), 397-404 (2020).

25. COVID-19 and Confinement: A Large-Scale French Survey of Social Challenges and Health INSERM. https://presse.inserm.fr/en/covid-19-and-confinement-a-large-scale-french-survey-of-socialchallenges-and-health/39099/ (2020).

26. Étude Longitudinale Française depuis l'Enfance. Ined - Institut national d'études démographiques. https://www.elfe-france.fr/ (2021). 
27. Charles, M. A. et al. Cohort Profile: The French national cohort of children (ELFE): birth to 5 years. Int J Epidemiol. 49(2), 368-369 (2020).

28. EPIPAGE 2: a preterm birth cohort in France in 2011. BMC Pediatrics. https://bmcpediatr.biomedcentral.com/articles/10.1186/1471-2431-14-97 (2021).

29. Carrat, F. et al. Incidence and Risk Factors of Illnesses Presumably Caused by A SARS-CoV-2 Infection in The General Population During The Lockdown Period: A Multi-Cohort Study. BMC Infectious Diseases. figshare 10.21203/rs.3.rs-90873/v1 (2020).

30. Goodman, R. Psychometric Properties of the Strengths and Difficulties Questionnaire. J Am Acad Child Adolesc Psychiatry. 40(11), 1337-1345 (2001).

31. Goodman, A. \& Goodman, R. Strengths and Difficulties Questionnaire as a Dimensional Measure of Child Mental Health. J Am Acad Child Adolesc Psychiatry. 48(4), 400-403 (2009).

32. Axford N. Defining and Classifying Children in Need. 590 (Routledge, 2017).

33. Hall, C. L. et al. The validity of the Strengths and Difficulties Questionnaire (SDQ) for children with ADHD symptoms. PLoS ONE. https://www.ncbi.nlm.nih.gov/pmc/articles/PMC6583960/ (2019).

34. Shojaei, T., Wazana, A., Pitrou, I,. \& Kovess, V. The strengths and difficulties questionnaire: validation study in French school-aged children and cross-cultural comparisons. Soc Psychiatry Psychiatr Epidemiol. 44(9), 740-747 (2009).

35. Thierry, X. et al. Les enfants à l'épreuve du premier confinement. Popul Soc. 585(1), 1-4 (2021).

36. Burgette, L. F. \& Reiter, J. P. Multiple Imputation for Missing Data via Sequential Regression Trees. Am J Epidemiol. 172(9), 1070-1076 (2010).

37. Zhao, Y. \& Long, Q. Variable Selection in the Presence of Missing Data: Imputation-based Methods. Wiley Interdiscip Rev Comput Stat. https://www.ncbi.nlm.nih.gov/pmc/articles/PMC5659333/ (2017).

38. Heymans, M. W., Van Buuren, S., Knol, D. L., Van Mechelen, W. \& De Vet, H. C. Variable selection under multiple imputation using the bootstrap in a prognostic study. BMC Med Res Methodol. 7, 33 (2007).

39. Zou, H. \& Hastie, T. Regularization and variable selection via the elastic net. J R Stat Soc Ser B Stat Methodol. 67(2), 301-320 (2005).

40. Mowlem, F. D. et al. Sex differences in predicting ADHD clinical diagnosis and pharmacological treatment. Eur Child Adolesc Psychiatry. 28(4), 481-489 (2019).

41. Barriuso-Lapresa, L. M., Hernando-Arizaleta, L. \& Rajmil, L. Reference values of the Strengths and Difficulties Questionnaire (SDQ) version for parents in the Spanish population, 2006. Actas Esp Psiquiatr. 42(2), 43-48 (2014).

42. Laurens, K. R. et al. The 2015 Middle Childhood Survey (MCS) of mental health and well-being at age 11 years in an Australian population cohort. BMJ Open.

https://www.ncbi.nlm.nih.gov/pmc/articles/PMC5726143/ (2017).

43. Hou, F., Bi, F., Jiao, R., Luo, D. \& Song, K. Gender differences of depression and anxiety among social media users during the COVID-19 outbreak in China: a cross-sectional study. BMC Public Health. 
20(1), 1648 (2020).

44. Thapar, A. Attention deficit hyperactivity disorder. The Lancet. 387 (10024), 1240-1250 (2016).

45. Banaschewski, T. et al. Attention-Deficit/Hyperactivity Disorder. Dtsch Ärztebl Int. 114(9), 149-159 (2017).

46. Faraone, S. V. et al. Attention-deficit/hyperactivity disorder. Nat Rev Dis Primer. 1(1), 1-23 (2015).

47. Montagna, A. \& Nosarti, C. Socio-Emotional Development Following Very Preterm Birth: Pathways to Psychopathology. Front Psychol. https://www.ncbi.nlm.nih.gov/pmc/articles/PMC4751757/ (2016).

48. Fountoulakis, K. N. et al. Self-reported changes in anxiety, depression and suicidality during the COVID-19 lockdown in Greece. J Affect Disord. 279, 624-629 (2021).

49. Franz, A. P. et al. Attention-Deficit/Hyperactivity Disorder and Very Preterm/Very Low Birth Weight: A Meta-analysis. Pediatrics. https://pediatrics.aappublications.org/content/141/1/e20171645 (2018)

50. Linnet, K. M. et al. Gestational age, birth weight, and the risk of hyperkinetic disorder. Arch Dis Child. 91(8), 655-660 (2006).

51. Wang, Z. H. et al. Prevalence of anxiety and depression symptom, and the demands for psychological knowledge and interventions in college students during COVID-19 epidemic: A large cross-sectional study. J Affect Disord. 275, 188-193 (2020).

52. Pedrosa, A. L. et al. Emotional, Behavioral, and Psychological Impact of the COVID-19 Pandemic. Front Psychol. https://www.ncbi.nlm.nih.gov/pmc/articles/PMC7561666/ (2020).

53. Islam, SMD-U., Bodrud-Doza, Md., Khan, R. M., Haque, MdA. \& Mamun, M. A. Exploring COVID-19 stress and its factors in Bangladesh: A perception-based study. Heliyon. 6(7), e04399 (2020).

54. Torales, J., O'Higgins, M., Castaldelli-Maia, J. M. \& Ventriglio, A. The outbreak of COVID-19 coronavirus and its impact on global mental health. Int J Soc Psychiatry. 66(4), 317-320 (2020).

55. Hvolby, A. Associations of sleep disturbance with ADHD: implications for treatment. Atten Deficit Hyperact Disord. 7(1), 1-18 (2015).

56. Cortese, S., Faraone, S. V., Konofal, E. \& Lecendreux, M. Sleep in children with attentiondeficit/hyperactivity disorder: meta-analysis of subjective and objective studies. J Am Acad Child Adolesc Psychiatry. 48(9), 894-908 (2009).

57. Sadeh, A., Pergamin, L. \& Bar-Haim, Y. Sleep in children with attention-deficit hyperactivity disorder: a meta-analysis of polysomnographic studies. Sleep Med Rev. 10(6), 381-398 (2006).

58. Reynaud, E., Forhan, A., Heude, B., Charles, M. A. \& Plancoulaine, S. Association of night-waking and inattention/hyperactivity symptoms trajectories in preschool-aged children. Sci Rep. 8(1), 15412 (2018).

59. Hoffman, J. A. \& Miller, E. A. Addressing the Consequences of School Closure Due to COVID-19 on Children's Physical and Mental Well-Being. World Med Health Policy. https://www.ncbi.nlm.nih.gov/pmc/articles/PMC7461306/ (2020).

60. Bahn, G. H. Coronavirus Disease 2019, School Closures, and Children's Mental Health. J Korean Acad Child Adolesc Psychiatry. 31(2), 74-79 (2020). 
61. Brown, W. J., Wilkerson, A. K., Boyd, S. J., Dewey, D., Mesa, F. \& Bunnell, B. E. A review of sleep disturbance in children and adolescents with anxiety. J Sleep Res. 27(3), e12635 (2018).

62. Quach, J. L., Nguyen, C. D., Williams, K. E. \& Sciberras, E. Bidirectional Associations Between Child Sleep Problems and Internalizing and Externalizing Difficulties From Preschool to Early Adolescence. JAMA Pediatr. 172(2), e174363 (2018).

63. Nunes, S., Campbell, M. K., Klar, N., Reid, G. J. \& Stranges, S. Relationships between sleep and internalizing problems in early adolescence: Results from Canadian National Longitudinal Survey of Children and Youth. J Psychosom Res. 139, 110279 (2020).

64. Hysing, M., Sivertsen, B., Garthus-Niegel, S. \& Eberhard-Gran, M. Pediatric sleep problems and socialemotional problems. A population-based study. Infant Behav Dev. 42, 111-118 (2016).

65. Catalano, R. et al. The Health Effects of Economic Decline. Annu Rev Public Health. 32(1), 431-450 (2011).

66. Golberstein, E., Gonzales, G. \& Meara, E. How do economic downturns affect the mental health of children? Evidence from the National Health Interview Survey. Health Econ. 28(8), 955-970 (2019).

67. Wathelet, M. et al. Factors Associated With Mental Health Disorders Among University Students in France Confined During the COVID-19 Pandemic. JAMA Netw Open.

https://www.ncbi.nlm.nih.gov/pmc/articles/PMC7584927/ (2020).

68. Moreno, C. et al. How mental health care should change as a consequence of the COVID-19 pandemic. Lancet Psychiatry. 7(9), 813-824 (2020).

69. Donovan, G. H., Michael, Y. L., Gatziolis, D., Mannetje, A. \& Douwes, J. Association between exposure to the natural environment, rurality, and attention-deficit hyperactivity disorder in children in New Zealand: a linkage study. Lancet Planet Health. 3(5), 226-234 (2019).

70. Knopf, H., Hölling, H., Huss, M. \& Schlack, R. Prevalence, determinants and spectrum of attentiondeficit hyperactivity disorder (ADHD) medication of children and adolescents in Germany: results of the German Health Interview and Examination Survey (KiGGS). BMJ Open. 2(6) (2012).

71. Amerio, A. et al. COVID-19 Lockdown: Housing Built Environment's Effects on Mental Health. Int J Environ Res Public Health. https://www.ncbi.nlm.nih.gov/pmc/articles/PMC7459481/ (2020).

72. Zarrabi, M., Yazdanfar, S-A. \& Hosseini S-B. COVID-19 and healthy home preferences: The case of apartment residents in Tehran. J Build Eng. 35, 102021 (2021).

73. Vandentorren, S. et al. Premiers résultats des facteurs associés à la résilience et à la santé mentale des enfants et des adolescents (9-18 ans) lors du premier confinement lié à la Covid-19 en France. Santé Publique France. /import/premiers-resultats-des-facteurs-associes-a-la-resilience-et-a-la-santementale-des-enfants-et-des-adolescents-9-18-ans-lors-du-premier-confinemen (2021).

74. Nekliudov, N. A. et al. Excessive Media Consumption About COVID-19 is Associated With Increased State Anxiety: Outcomes of a Large Online Survey in Russia. J Med Internet Res. 22(9), e20955 (2020).

75. Khan, M. I., Saleem, H. A. R., Anwar, M. F. \& Chang, Y-C. Novel Coronavirus and Emerging Mental Health Issues-A Timely Analysis of Potential Consequences and Legal Policies Perspective. Fudan J 
Humanit Soc Sci. 14(1), 87-105 (2021).

76. Fitzpatrick, K. M., Drawve, G. \& Harris, C. Facing new fears during the COVID-19 pandemic: The State of America's mental health. J Anxiety Disord. 75, 102291 (2020).

77. Ye, J. Pediatric Mental and Behavioral Health in the Period of Quarantine and Social Distancing With COVID-19. JMIR Pediatr Parent. https://www.ncbi.nIm.nih.gov/pmc/articles/PMC7389340/ (2020).

\section{Tables}

Table 1. Characteristics of children participating in SAPRIS project in France on weighted data $(n=5702)$. 







\begin{tabular}{|l|l|}
\hline No & $3175(60.2)$ \\
\hline Time per day spent (in hours) on: & \\
\hline Reading, drawing, board games & $2.37(0.06)$ \\
\hline Screen for recreation & $3.15(0.08)$ \\
\hline Social network & $0.09(0.02)$ \\
\hline Physical activity & $2.74(0.08)$ \\
\hline Children's schooling & \\
\hline School situation before lockdown & $5080(97.2)$ \\
\hline Normal & $188(2.8)$ \\
\hline With assistance or unschooled & \\
\hline Time devoted to schoolwork per day & $124(2.6)$ \\
\hline None, less than 1 hour or do not know & $2764(62.8)$ \\
\hline 1-3 hours & $1496(34.5)$ \\
\hline$>3$ hours & \\
\hline Screen time for educational reasons per day (in hours) & $0.83(0.04)$ \\
\hline Tutoring difficulty & $126(3.3)$ \\
\hline Tutoring with difficulty & $1003(22.5)$ \\
\hline Tutoring without difficulty & $3199(74.2)$ \\
\hline No tutoring & \\
\hline
\end{tabular}

Table 2. Sociodemographic and socioeconomic characteristics of children's families in SAPRIS project in France on weighted data $(n=5702)$. 


\begin{tabular}{|c|c|c|}
\hline & Mean (SD) & $\mathrm{n}(\%)$ \\
\hline \multicolumn{3}{|l|}{ Socioeconomic characteristics } \\
\hline \multicolumn{3}{|l|}{ Parents' occupational category ${ }^{1}$} \\
\hline Executive & & $1652(21.9)$ \\
\hline Intermediate and executive & & $1497(23.5)$ \\
\hline Intermediate and employee & & $1390(29.1)$ \\
\hline Independent & & $475(10.3)$ \\
\hline Laborer & & $347(9.2)$ \\
\hline Both inactive or only one employee/laborer & & $186(6.0)$ \\
\hline \multicolumn{3}{|l|}{ Change in parents' work situation ${ }^{2}$} \\
\hline Change at least for one parent & & $1483(43.9)$ \\
\hline No change for either parent & & $2763(56.1)$ \\
\hline \multicolumn{3}{|l|}{ Parents' distance-working ${ }^{3}$} \\
\hline Neither working & & $1973(45.6)$ \\
\hline One teleworking, the other not working & & $2013(28.1)$ \\
\hline At least one working outside & & $1214(21.8)$ \\
\hline Both teleworking & & $358(4.5)$ \\
\hline \multicolumn{3}{|l|}{ Perceived financial situation } \\
\hline Affluent and constant income & & $3143(50.6)$ \\
\hline Affluent and declining income & & $557(7.9)$ \\
\hline Modest and constant income & & $879(20.3)$ \\
\hline Modest and declining income & & $934(21.3)$ \\
\hline \multicolumn{3}{|l|}{ Housing conditions } \\
\hline \multicolumn{3}{|l|}{ Type of housing } \\
\hline Rural house & & $2299(36.5)$ \\
\hline Urban house & & $2235(30.9)$ \\
\hline Urban flat with balcony/garden & & $714(23.3)$ \\
\hline Urban flat without balcony/garden & & $218(6.6)$ \\
\hline Other & & $84(2.8)$ \\
\hline
\end{tabular}




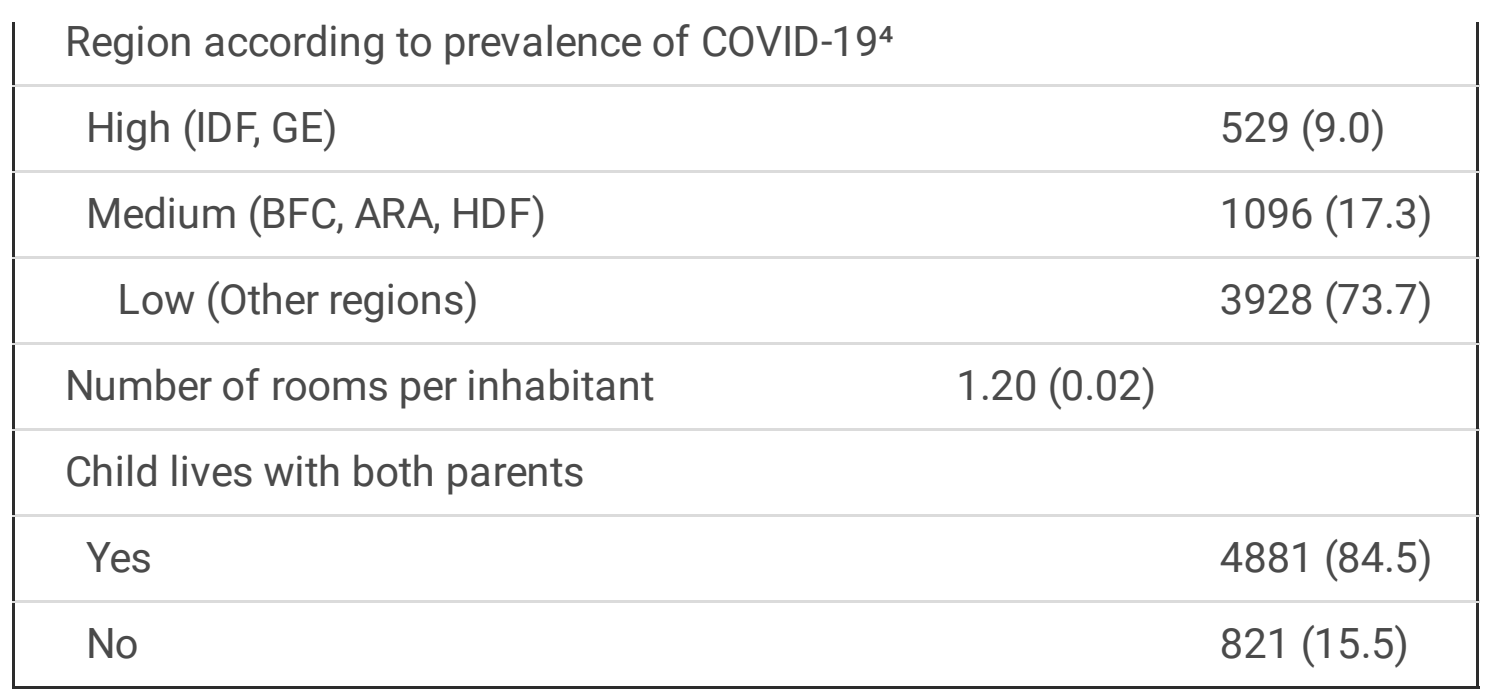

${ }^{1}$ In the absence of a spouse, the respondent who is executive refers to «intermediate and executive» category; which is intermediate corresponds to «intermediate and employee» category; and which is employee, worker or without profession refers to «both inactive or only one employee/labourer» category.

${ }^{2}$ In the absence of a spouse, the respondent who has undergone a change in work situation during the lockdown refers to «change at least for one parent» category and the one who has not undergone any change to «no change for either parent» category.

IIn the absence of a spouse, the respondent who is not working is associated with the "neither working» category; the one who teleworks refers to «both teleworking» category; and the respondent who works outside to «at least one working outside» category.

${ }^{4}$ Region classified according to the prevalence of COVID-19 at the beginning of the epidemic (High: lle de France and Grand Est vs Medium: Bourgogne-Franche-Comté, Auvergne-Rhône-Alpes and Hauts-deFrance vs Low: other regions)

Table 3. Factors associated with hyperactivity/inattention: multinomial logistic regressions adjusted on prematurity $(n=5097)$ 
Hyperactivity/inattention

Abnormal

vs normal

Boundary state vs normal

Sex (Female: ref. group)

Male

2.05[1.73;

$2.44]$

$1.61[1.32 ; 1.97]$

Chronic disease (No: ref. group)

Yes

$3.68[2.78 ;$

4.88]

$1.68[1.11 ; 2.55]$

Born pre-term (No: ref. group)

Yes

$1.71[1.41$

2.07]

$1.44[1.14 ; 1.81]$

Access to specialized care prior to COVID-19 (No: ref. group)

Yes and pursuit during school closures

$2.48[1.62$

$3.81]$

Yes but no pursuit during school closures

$2.40[1.99 ;$

2.89]

Covid cases in household (No: ref. group)

Yes

$1.03[0.68$

$1.55]$

$1.14[0.72 ; 1.81$

$2.31[1.41 ; 3.77]$

$1.56[1.22 ; 1.97]$

\section{Children's mental health}

Emotional symptoms (Normal: ref. group)

Abnormal

$5.73[4.54 ;$

7.24]

$1.75[1.21 ; 2.53]$

Boundary state

$2.84[2.09$;

$3.85]$

$2.57[1.82 ; 3.62]$

\section{Children's health behavior}

Sleeping difficulties (No: ref. group)

Yes

$2.68[2.26$

3.17]

$1.88[1.54 ; 2.28]$

\section{Children's schooling}

School situation before lockdown (Normal: ref. group)

With assistance or unschooled

$3.85[2.74 ; \quad 1.84[1.12 ; 3.01$
$5.40]$


Time devoted to schoolwork per day (None, less than 1 hour: ref. group)
1-3 hours
$0.51[0.33$
$0.78[0.42 ; 1.47]$
$>3$ hours
$0.52[0.33$
$0.82]$
$0.91[0.48 ; 1.73]$

Tutoring (Tutoring without difficulty: ref. group)

Tutoring with difficulty

$3.92[3.25$

4.73]

No tutoring

$2.96[1.85$;

$1.95[1.10 ; 3.44]$

4.72]

\section{Socio-economic characteristics}

Parents' occupational category (Executive: ref. group)

Intermediate and executive

$1.34[1.06$

1.70]

$0.90[0.68 ; 1.18]$

Intermediate and employee

$1.58[1.24$

$1.28[0.99 ; 1.66]$

Independent

$1.45[1.05$

$2.01]$

$0.95[0.64 ; 1.42]$

Both inactive or only one employee/laborer

$1.15[0.77$

$1.71]$

$1.07[0.70 ; 1.65]$

Laborer

$3.03[2.05 ;$

4.48]

$1.43[0.84 ; 2.42]$

Change in parents' work situation (No change for either parent: ref. group)

Change at least for one parent $1.33[1.11$ 1.59]

Parents' distance-working (No one working: ref. group)

At least one working outside

$0.73[0.60$

$0.89]$

$0.94[0.74 ; 1.18]$

Both teleworking

$0.78[0.63$

$0.98]$

$0.83[0.63 ; 1.09]$

One teleworking and the other not working
$0.73[0.51$
$0.80[0.52 ; 1.24]$
$1.06]$

Perceived financial situation (Affluent and constant income: ref. group)

Affluent and declining income

$1.19[0.89$

$1.60]$

$1.47[1.07 ; 2.02]$ 
Modest and constant income

$1.52[1.21$

$1.91]$

$1.29[0.98 ; 1.71]$

Modest and declining income

$2.01[1.62$

2.49]

$1.82[1.41 ; 2.35]$

\section{Sociodemographic information concerning housing} conditions

Type of housing (Rural house: ref. group)

Urban house

$0.77[0.64$

$0.94]$

Urban flat with balcony/garden

$1.04[0.80$

$1.34]$

Urban flat without balcony/garden

$1.32[0.89 ;$

1.94]

Other

$1.34[0.70$;

2.56]

$1.07[0.86 ; 1.33]$

$1.26[0.93 ; 1.71]$

$0.83[0.46 ; 1.49]$

$2.65[1.43 ; 4.91]$

Regions affected by COVID (Low: ref. group) ${ }^{1}$

High (IDF, GE)

$0.93[0.69 ;$

1.26]

Medium (BFC, ARA, HDF)

$0.98[0.79$;

$1.21]$

$1.14[0.90 ; 1.46]$

$0.80[0.63$

1.00]

$1.21[0.88 ; 1.68]$

Number of rooms per inhabitant

Child living with both parents (Yes: ref. group)

No

$1.53[1.24$

$1.18[0.90 ; 1.55]$

1.90]

${ }^{1}$ Region classified according to prevalence of COVID-19 at beginning of epidemic (High: lle de France and Grand Est vs Medium: Bourgogne-Franche-Comté, Auvergne-Rhône-Alpes and Hauts-de-France vs Low: other regions)

Table 4. Factors associated with emotional symptoms: multinomial logistic regressions adjusted on prematurity $(n=5097)$ 
Emotional symptoms

Abnormal

vs normal

Boundary state vs normal

Sex (Female: ref. group)

$$
\text { Male }
$$

$0.81[0.66$

$0.73[0.57 ; 0.93]$

$1.00]$

Chronic disease (No: ref. group)

Yes

$2.56[1.81$

$3.63]$

Born pre-term (No: ref. group)

Yes

$1.62[1.28$

2.06]

$1.25[0.94 ; 1.68]$

Access to specialized care prior to COVID-19 (No: ref. group)

Yes and pursuit during school closures

$2.20[1.31$

3.68]

$2.00[1.29 ; 3.11]$

$2.01[1.59$;

$2.55]$

Yes but no pursuit during school closures

$1.66[1.08$

$2.56]$

$1.18[0.66 ; 2.10]$

Yes

$6.29[4.93$

8.03]

$2.12[1.17 ; 3.84]$

$1.69[1.28 ; 2.24]$

Covid cases in household (No: ref. group)

\section{Children's health behavior}

Sleeping difficulties (No: ref. group)

Yes

$1.69[1.07$

2.68]

$3.15[2.46 ; 4.04]$

\section{Children's schooling}

School situation before lockdown (Normal: ref. group)

With assistance or unschooled

Time devoted to school work per day (None, less than 1

hour: ref. group)

1-3 hours

$0.78[0.41$

$1.48]$

$>3$ hours

$0.86[0.45$

$1.63]$

$1.61[0.92 ; 2.81]$ 
Tutoring with difficulty

No tutoring
$1.99[1.58$

2.52]

$1.81[1.02$

3.22]
$2.12[1.62 ; 2.78]$

$1.38[0.64 ; 2.98]$

\section{Socio-economic characteristics}

Parents' occupational category (Executive: ref. group)

Intermediate and executive

Intermediate and employee

Independent

Both inactive or only one employee/laborer

Laborer
$1.72[1.27$

2.34]

$1.73[1.27$

2.36]

$1.71[1.13$

2.60]

$1.92[1.22$ 3.01]

$3.23[1.99 ;$

$5.24]$
$1.21[0.88 ; 1.68]$

$1.23[0.88 ; 1.71]$

$0.90[0.54 ; 1.51]$

$0.80[0.43 ; 1.49]$

$1.66[0.90 ; 3.07]$

Change in parents' work situation (No change for either parent: ref. group)

Change at least for one parent 1.46 [1.17;

1.82]

$1.13[0.87 ; 1.47]$

Parents' distance-working (neither working: ref. group)

At least one working outside

$0.73[0.57$

$0.94]$

Both telework

$0.82[0.62$ 1.09]

One teleworks and the other does not work

$0.82[0.51$

1.29]

Perceived financial situation (Affluent and constant income: ref. group)

Affluent and declining income

$0.89[0.59$

1.34]

Modest and constant income

$1.73[1.32$

2.27]

Modest and declining income

$1.96[1.50$

2.55]

$0.99[0.74 ; 1.32]$

$0.96[0.69 ; 1.34]$

$1.17[0.72 ; 1.90]$

$1.49[1.06 ; 2.09]$

$2.14[1.58 ; 2.89]$

\section{Sociodemographic information's concerning housing} conditions

Type of housing (Rural house: ref. group) 


\begin{tabular}{|lll|}
\hline Urban house & $0.79[0.63 ;$ & $1.01[0.76 ; 1.34]$ \\
& $1.00]$ & \\
\hline Urban flat with balcony/garden & $0.96[0.69 ;$ & $1.69[1.20 ; 2.38]$ \\
& $1.34]$ & \\
Urban flat without balcony/garden & $1.62[1.03 ;$ & $1.43[0.79 ; 2.31]$ \\
& $2.54]$ & \\
\hline Other & $0.97[0.41 ;$ & $1.36[0.53 ; 3.46]$ \\
& $2.26]$ & \\
\hline Regions affected by COVID (Low: ref. group) & & \\
\hline High (IDF, GE) & $1.24[0.88 ;$ & $0.71[0.45 ; 1.14]$ \\
& $1.75]$ & \\
\hline Medium (BFC, ARA, HDF) & $1.11[0.86 ;$ & $0.75[0.54 ; 1.05]$ \\
& $1.44]$ & \\
\hline Number of rooms per inhabitant & $0.96[0.77 ;$ & $0.76[0.54 ; 1.07]$ \\
& $1.19]$ & \\
\hline Child living with both parents (Yes: ref. group) & & $1.35[1.03 ;$ \\
\hline No & $1.77]$ & $1.18[0.85 ; 1.64]$ \\
\hline
\end{tabular}

${ }^{1}$ Region classified according to prevalence of COVID-19 at beginning of epidemic (High: lle de France and Grand Est vs Medium: Bourgogne-Franche-Comté, Auvergne-Rhône-Alpes and Hauts-de-France vs Low: other regions)

Table 5. Associations between most predictive factors from elastic net selection and hyperactivity/inattention symptoms: multivariate multinomial logistic regressions $(n=5097)$ 
Hyperactivity/inattention ${ }^{1}$

Abnormal vs normal

Boundary state vs normal

Sex (Female: ref. group)

Male

$2.29[1.90 ; 2.76]$

$1.75[1.42 ; 2.14]$

Access to specialized care prior to COVID-19 (No: ref. group)

Yes and pursuit during school closures

Yes but no pursuit during school closures

\section{Children's mental health}

Emotional symptoms (Normal: ref. group)

Abnormal

Boundary state

\section{Children's health behavior}

Sleeping difficulties (No: ref. group)

Yes

$2.05[1.70 ; 2.47]$
$1.80[1.08 ; 2.99]$

$1.27[0.99 ; 1.64]$

\begin{tabular}{ll}
$1.42[0.87 ; 2.32]$ & $1.80[1.08 ; 2.99]$ \\
\hline $1.51[1.21 ; 1.88]$ & $1.27[0.99 ; 1.64]$
\end{tabular}

$1.51[1.21 ; 1.88]$
$4.06[3.11 ; 5.29] \quad 1.43[0.97 ; 2.09]$

$2.14[1.54 ; 2.97] \quad 2.11[1.47 ; 3.01]$

\section{Children's schooling}

School situation before lockdown (Normal: ref. group)

With assistance or unschooled

$2.13[1.43 ; 3.17] \quad 1.24[0.73 ; 2.10]$

Time devoted to schoolwork per day (Nonelless than 1 hour: ref. group)

1-3 hours

$0.55[0.34 ; 0.91]$

$0.85[0.45 ; 1.60]$

$>3$ hours

$0.56[0.33 ; 0.92]$

$0.98[0.51 ; 1.87]$

Tutoring (Tutoring without difficulty: ref. group)

Tutoring with difficulty

No tutoring
$3.25[2.64 ; 3.99]$

$2.47[1.48 ; 4.11]$
$2.04[1.61 ; 2.57]$

$1.83[1.02 ; 3.31]$

\section{Socio-economic characteristics}

Parents' occupational category (Executive: ref. group)

Intermediate and executive

Intermediate and employee

Independent
$1.20[0.94 ; 1.55] \quad 0.87[0.66 ; 1.14]$

$1.25[0.97 ; 1.62] \quad 1.18[0.90 ; 1.55]$

$1.26[0.88 ; 1.80] \quad 0.92[0.61 ; 1.39]$ 


\begin{tabular}{|c|c|c|}
\hline Both inactive or only one employee/laborer & $0.89[0.58 ; 1.38]$ & $1.01[0.64 ; 1.57]$ \\
\hline Laborer & $1.66[1.01 ; 2.71]$ & $1.11[0.61 ; 2.03]$ \\
\hline \multicolumn{3}{|l|}{ Housing conditions } \\
\hline \multicolumn{3}{|l|}{ Type of housing (Rural house: ref. group) } \\
\hline Urban house & $0.79[0.64 ; 0.97]$ & $1.06[0.84 ; 1.33]$ \\
\hline Flat with balcony/garden & $0.95[0.71 ; 1.26]$ & $1.17[0.85 ; 1.60]$ \\
\hline Flat without balcony/garden & $1.05[0.67 ; 1.64]$ & $0.74[0.40 ; 1.36]$ \\
\hline Other & $1.07[0.53 ; 2.17]$ & $2.28[1.20 ; 4.35]$ \\
\hline \multicolumn{3}{|l|}{ Child living with both parents (Yes: ref. group) } \\
\hline No & $1.21[0.92 ; 1.59]$ & $1.06[0.77 ; 1.45]$ \\
\hline
\end{tabular}

${ }^{1}$ Adjusted on sex, prematurity, access to specialized care prior to COVID-19 and its continuation during school closures, emotional symptoms, sleeping difficulties, school situation before lockdown, time devoted to schoolwork, tutoring, parents' occupational category, type of housing, and child living with both parents.

Table 6. Associations between most predictive factors from elastic net selection and emotional symptoms: multivariate multinomial logistic regressions $(n=5097)$ 
Emotional symptoms ${ }^{2}$

Abnormal

vs normal

Boundary state vs normal

Born pre-term (No: ref. group)

Yes

$1.34[1.03$

$1.73]$

$1.13[0.83 ; 1.53]$

COVID cases in household (No: ref. group)

Yes

$1.72[1.08$

2.73]

$1.14[0.63 ; 2.05]$

\section{Children's mental health}

Hyperactivity/inattention (Normal: ref. group)

Abnormal

$4.18[3.27$

$5.34]$

Boundary state

1.37[0.94;
2.00]

$2.12[1.50 ; 3.01]$

Children's health behavior

Sleeping difficulties (No: ref. group)

Yes

5.34 [4.16;
$6.86]$

$2.79[2.17 ; 3.60]$

\section{Socio-economic characteristics}

Parents' occupational category (Executive: ref. group)

Intermediate and executive

$1.61[1.16$

2.21]

Intermediate and employee

$1.38[0.97$

1.94]

Independent

$1.48[0.94$

$2.34]$

Both inactive or only one employee/laborer

$1.63[0.99 ;$

$2.66]$

Laborer

$1.67[0.97$

2.88]

$1.15[0.82 ; 1.60]$

$1.03[0.72 ; 1.48]$

$0.72[0.42 ; 1.23]$

$0.66[0.35 ; 1.27]$

$1.04[0.54 ; 2.01]$

Change in parents' work situation (No change for either parent: ref. group)

Change at least for one parent

1.17[0.91;
$1.51]$

$0.93[0.70 ; 1.25]$

Perceived financial situation (Affluent and constant 
income: ref. group)

Affluent and declining income

$0.79[0.51$

$1.22]$

$1.29[0.84 ; 1.98]$

Modest and constant income

$1.45[1.07$;

1.96]

Modest and declining income

$1.36[1.01 ;$

$1.84]$

$1.51[1.05 ; 2.15]$

$2.03[1.45 ; 2.84]$

${ }^{1}$ Adjusted on prematurity, COVID cases in household, hyperactivity/inattention symptoms, sleeping difficulties, parents' occupational category, change in parents' work situation and perceived financial situation. 